\title{
Sustentabilidad asociada al traspaso generacional en un tambo
}

\author{
Novaira $\mathrm{B}^{1^{*}}$, Gimenez $\mathrm{G}^{1,2,3}$, Marini $\mathrm{PR}^{1,3,4}$ \\ ${ }^{1}$ Centro Latinoamericano de Estudios de Problemáticas Lecheras (CLEPL). \\ ${ }^{2}$ Agencia de Extensión Rural, Instituto Nacional de Tecnología Agropecuaria, Roldán, Argentina. \\ ${ }^{3}$ Universidad Nacional de Rosario (UNR), Facultad de Ciencias Veterinarias, Casilda, Argentina. \\ ${ }^{4}$ Carrera del Investigador Científico de la UNR, Casilda, Argentina.
}

* Correspondencia: Bruno Novaira. CLEPL. Ovidio Lagos y Ruta 33 CP (2170) Casilda Provincia de Santa Fe, Argentina. E-mail: bnovaira@gmail.com

Recibido: 15 Noviembre 2020. Aceptado: 17 Mayo 2021. Disponible en línea: 16 Junio 2021

Editor: P. Beldomenico

RESUMEN. El enfoque de un sistema productivo tanto en la dinámica económica-productiva, socio-cultural y ambiental de la sustentabilidad brinda una mirada integral de las problemáticas estableciendo las limitantes y potencialidades con las que cuenta el sistema, las cuales tienen un impacto directo sobre la productividad. La continuidad de un emprendimiento a lo largo de las generaciones presenta dificultades desde el punto de vista empresarial y sistémico. Los desafíos humanos y técnicos traen aparejados conflictos de difícil resolución que en muchas ocasiones terminan con la disolución de la empresa o una fuerte modificación en su dirección para mejorar los resultados en los pilares mencionados.

SUMMARY. Sustainability associated with generational transfer in dairy farm. The focus of a production system on the economic, productive, socio-cultural and environmental dynamics of sustaina-bility offers a comprehensive perspective of common issues, establishing the limitations and potentialities of said system, which will impact productivity directly. Continuity of an enterprise over generations proves difficult from an entrepreneurial and systemic point of view. Human and technical challenges bring about complex conflicts which end in the dissolution of the enterprise in many instances or a considerable shift in direction in order to improve the results of the mentioned pillars.

Palabras clave: lechería, traspaso generacional, proceso productivo

Keywords: dairy, generational transfer, production process

\section{Introducción}

En el año 1989, por herencia de sus padres y luego de la división entre hermanos, un profesional de las ciencias agrarias adquirió una superficie de 100 hectáreas convirtiéndose en productor agropecuario. Los primeros años realizó agricultura, cría e invernada. Ante los resultados negativos que obtenía en esas actividades, su esposa le sugirió emprender en el negocio de la producción lechera (1994), con el objetivo de tener un ingreso mensual y generar mayor estabilidad económica a su familia (compuesta por ellos dos y tres hijos a cargo). Así nació el tambo Namuncurá. A lo largo de los años el tambo fue creciendo en carga animal y producción con altibajos, pero aun así, permitió al productor seguir invirtiendo en el sistema productivo mejorando su eficiencia. En el año 2012 ingresó a la empresa su primer hijo, también Ingeniero Agrónomo.
La empresa presentaba deudas y un bajo resultado económico hasta el año 2016, en que comenzó a recuperarse económicamente al punto de salir de sus deudas.

En 2017, el fallecimiento de la esposa del productor provocó una redistribución de las responsabilidades entre los integrantes de la familia, incorporándose hacia fines de ese año, el segundo hijo, profesional de la medicina veterinaria. En la actualidad, la empresa ha dejado de ser unipersonal, constituyéndose en una sociedad, dentro de la cual se están restableciendo los roles de los cuatro socios que la componen, encargándose los dos hijos del seguimiento técnico del establecimiento, el padre de familia de la administración y pormenores diarios; aunque la hija menor no tiene una participación directa en el manejo del establecimiento, está informada de lo que sucede en la 
empresa y manifiesta cierto interés en participar en un futuro.

Este estudio introduce a la evaluación de la sustentabilidad en su sentido más amplio (abarcando las dimensiones productiva, económica, social y ambiental) y cómo la mecánica del traspaso generacional ha incidido en algunos indicadores seleccionados de aquella.

En la Argentina el $75 \%$ de las empresas son familiares, representan el $30 \%$ del Producto Bruto Interno (PBI) y producen la mayoría de los bienes que se consumen en el país (Suárez et al., 2019). Una situación similar ocurre en el sector agropecuario, sin embargo, apenas el 30\% de las empresas agropecuarias superan el traspaso de la primera a la segunda generación, la mayoría desaparecen y las menos se venden, y solo siete de cada cien logran llegar a la tercera generación (Kaplun, 2012).

Esta elevada tasa de disolución obedece a distintas razones, entre las cuales se pueden citar las dificultades de sucesión del mando, la falta de profesionalización de sus componentes y la insuficiencia económica como medio de vida para todos los integrantes en el largo plazo. El período más peligroso que enfrenta una empresa es la sucesión, cuando la propiedad y la autoridad pasan de una persona a otra. En una empresa familiar, especialmente que pasa de la primera a la segunda generación, generalmente se cambia de una persona a varias; y eso es un desafío tanto para la empresa como para la familia. Se estima que el $85 \%$ de las crisis en una empresa familiar se dan alrededor de cómo resolver la sucesión. La autoridad y el liderazgo de una empresa pueden pasar a miembros de la generación siguiente, representantes de esa generación, o agentes externos (Eurichi y Suero, 2012). La familia tiene que darse cuenta de que la sucesión no es simplemente una decisión de negocios, familiar o financiera, sino un proceso que debe iniciarse con anticipación y durante muchos años, involucrando a todo el conjunto familia y empresa, que siempre requieren un delicado equilibrio (Eurichi y Suero, 2012).

Existen muchas razones por las cuales las empresas familiares no logran sobrevivir. Un estudio sobre micro y pequeñas empresas mexicanas, elaborado por Maza y Páez (1991), da cierta luz sobre las causas de su desaparición. Las causas señaladas por estos autores pueden dividirse en dos grandes grupos: las estructurales (que tienen que ver con la empresa) y las del entorno (que contemplan aspectos del mercado, financiamiento e impuestos) (Bastos et al., 2016).

Los factores administrativos son la principal causa de desaparición de las empresas familiares, seguida por las cuestiones de orden financiero y de política fiscal. Entre los factores administrativos que más influyen en la desaparición de las empresas familiares están la carencia o inadecuada utilización de los sistemas administrativos, el manejo ineficiente de los recursos y la falta de control (Belausteguigoitía, 2003). Por otra parte, de acuerdo con los estudios realizados en el 2002 y 2012 por el Grupo de Investigación en Empresa Familiar de INALDE Business School, se identificaron las principales dificultades de las empresas familiares en América Latina, encontrándose entre ellas las relacionadas con el ámbito familiar, la falta de un relevo generacional exitoso, debilidades en el ámbito patrimonial y empresarial (Gómez, 2013). Inclusive, muchas empresas familiares enfrentan dificultades por la falta de personal calificado, carencia de altruismo y una tendencia a la aversión al riesgo (Kets de Vries, 1996).

En este contexto, el traspaso generacional es un proceso con una dinámica particular, construido a partir de los valores de quienes lo componen, que incluyen el arte y conocimiento de "cómo" hacer las cosas. Esto hace la diferencia a la hora de gestionar sistemas complejos que incluyen la biología y el clima. En tal sentido, Viglizzo (2001) aporta una idea sobre la incorporación de conocimiento y tecnología en el tiempo y el espacio en cada empresa rural. En cada onda de acumulación de conocimiento es necesario lograr una intervención en los procesos para lo cual es necesario incorporar la tecnología de procesos, que consiste en la utilización de los recursos disponibles de manera eficiente para lograr un alto impacto en el sistema con bajo costo de insumos. Se realiza generando una fuerte relación de vínculos entre las personas que intervienen en el sistema visualizando objetivos comunes que representan un beneficio para el equipo de trabajo en su conjunto impactando sobre las tres dimensiones del sistema productivo.

El objetivo del trabajo fue analizar el efecto de la incorporación paulatina de nuevos profesionales familiares, en la sustentabilidad de un sistema de producción de bovinos para leche.

\section{Materiales y métodos}

El establecimiento se encuentra ubicado a 10 kilómetros de la localidad de Centeno (32 $12^{\prime} 22^{\prime \prime S}$ $61^{\circ} 23^{\prime} 43^{\prime \prime} 0$ ), el mismo cuenta con 90 hectáreas propias y 60 alquiladas. Se encuentra conformado por 12 lotes destinados a la producción de forrajes y verdeos para la alimentación de vacas Holando Argentino (HA).

Se caracteriza el sistema comparando utilizando una herramienta generada desde el Programa Nacional de Producción Animal (PNPA 1126043) INTA (Gimenez et al., 2018), la cual se aplicó a tres ejercicios productivos específicos: (2012-2013 Propietario); (2014-2015 Propietario e Hijo) y (2017-2018 Propietario e hijos). Se comparan los resultados obtenidos sobre las distintas 
dimensiones de sustentabilidad durante los tres períodos.

Se tomaron tres períodos anuales desde el 1 de julio al 30 de junio del año siguiente con el objetivo de analizar el efecto de la incorporación paulatina de nuevos profesionales familiares a lo largo de seis años evaluando indicadores de sustentabilidad (económico- productivos, sociales y ambientales) en un sistema de producción de bovinos para leche.

Los períodos analizados se encuentran separados por más de un año teniendo en cuenta la incorporación de los hijos del productor.

En ocasiones vale la pena analizar cortes sincrónicos (escenarios), pero siempre teniendo en cuenta la historia completa del objeto de estudio (Giri, 2015).

\section{Recursos humanos y roles dentro del establecimiento actualmente}

La estructura de los recursos humanos del estable-cimiento se describe en el siguiente diagrama y descripción asociada:

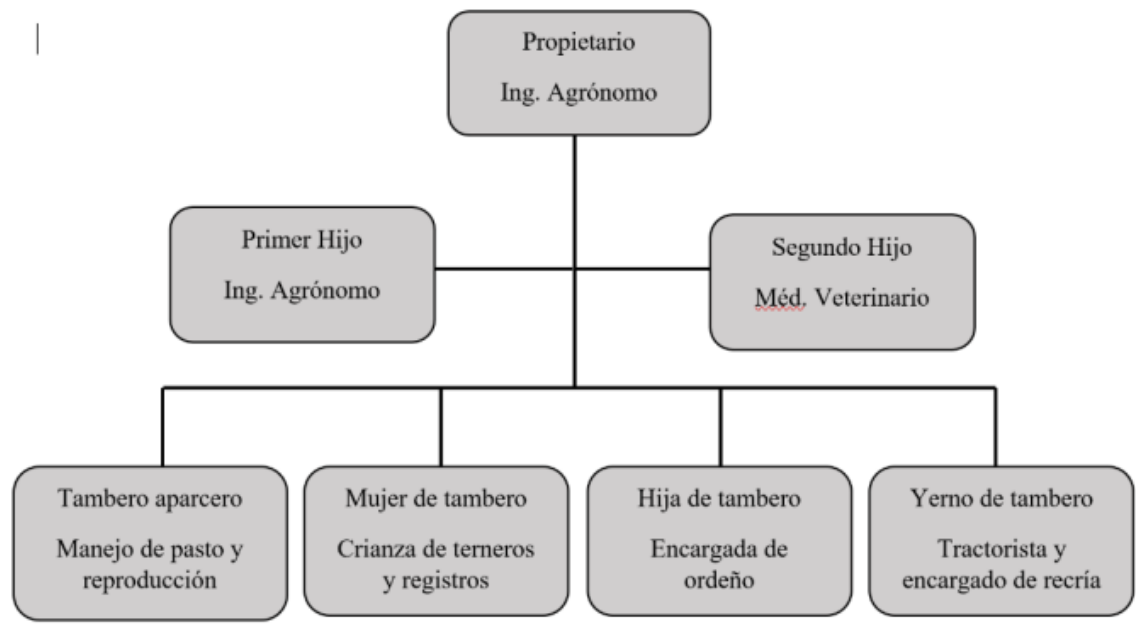

Propietario: está a cargo de la administración general de establecimiento.

Primer hijo (Ing. Agrónomo): planificación de rotaciones, siembra de pasturas, cultivos y de la dieta del módulo de recrías y preparto.

Segundo hijo (Méd. Veterinario): gestiona la crianza artificial de neonatos, la sanidad de todo el rodeo y el monitoreo reproductivo del rodeo.

Tambero aparcero o asociativo : manejo de las vacas a campo tanto rodeo de ordeño como vacas secas. El contrato asociativo de explotación tambera es de naturaleza agraria, que configura una particular relación participativa.

Esposa de Tambero: registra todos los eventos (partos nacimientos, abortos, muertes, ventas, servicios, secados, tratamientos).
Hija de Tambero: la rutina de ordeño y por lo tanto de controlar la limpieza tanto de los sectores como del equipo de ordeño.

Tractorista (yerno de tambero): es responsable de racionar con concentrado diariamente a toda la recría, el preparto y suministrar rollos en todas las categorías incluyendo vacas de ordeño y secas.

Los francos son repartidos cada 15 días, dividiendo el personal en dos grupos, quedando uno de guardia, otro de franco y viceversa, los fines de semana no se incluyen tareas extras, solo se realiza una rutina mínima, ordeño, distribución de concentrado en recría y leche a los terneros menores de sesenta días. 


\section{Variables analizadas}

Para la evaluación de la sustentabilidad del sistema se utilizó una herramienta desarrollada por el Programa Nacional de Producción Animal (PNPA 1126043) del Instituto Nacional de Tecnología Agropecuaria (INTA) (Litwin et al., 2016). Los rangos están definidos por por esa herramienta y se le asignó colores para identificar resultados. Deseables: verde, Regulares: amarillo e Indeseables rojo La misma consta de una serie de planillas basadas en Microsoft Excel $^{\circledR}$, en las cuales recolecta datos del establecimiento (generales, rodeo, producción de leche, instalaciones, manejo alimentario, planteo técnico), que luego son utilizados para calcular el valor de un set de indicadores de sustentabilidad económico-productiva, socio-cultural y ecológicoambiental. De todos ellos se seleccionaron una cantidad limitada de cada dimensión, a saber:

La dimensión económico-productiva se analizó con los indicadores que muestra la Tabla 1 y que se describen a continuación

Productividad ajustada: Se calcula como los kilos de leche producidos anualmente por hectárea dedicada a las vacas totales (VT: vacas en ordeño + vacas secas). Al igual que todos los indicadores que involucren la producción de leche, debe expresarse como leche corregida al $4 \%$ de grasa butirosa y 3,3 \% de proteína bruta. Para poder estandarizar las evaluaciones de tambos de distintas regiones se propone corregir el valor del indicador ajustándolo por el índice de productividad (IP) de la tierra. Este índice expresa la influencia conjunta de los factores de suelos sobre la productividad de cultivos, en el cual se asigna a cada factor de suelo un porcentaje de un valor ideal para luego multiplicarlos. Refleja numéricamente la capacidad actual de un suelo para obtener un rendimiento en un determinado cultivo, independientemente de los factores económicos que puedan condicionar el desarrollo de ciertas orientaciones productivas.

Crecimiento del rodeo: Se calcula como la cantidad de vaquillonas propias que ingresan al tambo y las que son vendidas que exceden la necesidad de reposición. La necesidad de reposición se calcula como la suma del descarte + mortandad de vacas.

Porcentaje del gasto de alimentación de las vacas totales en relación al ingreso bruto: tiene en cuenta que el gasto de alimentación es el principal componente del costo de un tambo. Los costos directos se estimaron a partir del costo directo de alimentación, que representa en promedio un 64,4\% del costo directo total (Roulet, 2013; Agromercado, 2014; Centeno y Almada, 2019).

La dimensión socio-cultural se analizó con los indicadores que muestra la Tabla 2 y que se describen a continuación.
Calidad de vida del entorno: Se trata de un indicador compuesto, que tiene en cuenta características objetivas como las distancias para acceder a poblados, centros educacionales y de salud, y la disponibilidad de servicios básicos como electricidad y telefonía.

Sucesión: Este indicador tiene en cuenta la predisposición para continuar la actividad de algunos de los integrantes de la familia.

Formación: tiene en cuenta los cursos que ha realizado el productor, las charlas a las que ha asistido y la importan-cia que le da a la capacitación de los recursos humanos vinculados con el establecimiento.

La dimensión ecológico-ambiental se analizó con los indicadores que muestra la Tabla 3 y que se describen a continuación.

Riesgo de contaminación de aguas subterráneas: Considera la probabilidad del impacto en las reservas acuosas como consecuencia del ingreso al sistema de insumos (fertilizantes, plaguicidas, alimentos, drogas veterinarias) que aporten nutrientes en exceso o sustancias biocidas. Tiene en cuenta la distancia entre perforaciones con relación a los puntos de mayor probabilidad de ocurrencia de estos aportes.

Manejo de los efluentes: Se consideran aspectos de planificación, infraestructura y manejo, ya que importa la previsión sobre el lugar al cual se realiza el vertido de los efluentes, los sistemas de tratamiento instalados en el establecimiento y las buenas prácticas destinadas a evitar la contaminación interna o al exterior del predio.

Emisión de gases con efecto invernadero (GEI): Las actividades agropecuarias pueden incrementar la concen-tración atmosférica de GEl, cuya tasa de emisión se calcula en base a los parámetros propuestos por el Calculador para tambos de AACREA (2011), que considera emisiones de materias primas, tambo $\mathrm{y}$ fábrica. 
Tabla 1. Indicadores de la dimensión económico-productiva.

\begin{tabular}{|c|c|c|c|c|c|}
\hline Indicador & Componente & \multicolumn{4}{|c|}{ Escala de valoración } \\
\hline & & Unidad & Verde & Amarillo & Rojo \\
\hline $\begin{array}{l}\text { Productividad/índice de } \\
\text { productividad del suelo } \\
\text { (IP) }\end{array}$ & Productividad ajustada & $\begin{array}{c}\mathrm{Kg} \\
\text { leche/haVT/año/IP }\end{array}$ & $>9.288$ & $9.288-7.224$ & $<7.224$ \\
\hline Crecimiento del rodeo & $\begin{array}{l}\text { Vaquillonas propias que } \\
\text { ingresan al tambo por } \\
\text { sobre la necesidad de } \\
\text { reposición }\end{array}$ & $\%$ & $>5$ & $5-0,1$ & 0 \\
\hline $\begin{array}{l}\text { Margen económico del } \\
\text { tambo }\end{array}$ & $\begin{array}{l}\% \text { gasto alimentación } \\
\text { VT/IB }\end{array}$ & $\%$ & $<40$ & $40-60$ & $>60$ \\
\hline
\end{tabular}

Tabla 2. Indicadores de la dimensión económico-productiva. Los rangos están definidos por INTA (Litwin et al., 2016) y se le asignó colores para identificar resultados. Deseables: verde, Regulares: amarillo, e Indeseables rojo. Referencias: (1) Transitabilidad. Buena: asfalto hasta la entrada o menos de $3 \mathrm{~km}$ por camino mejorado o menos de $1 \mathrm{~km}$ de tierra. Regular: 3-5 km por camino mejorado o 1-3 $\mathrm{km}$ por tierra a entrada. Mala: más de $5 \mathrm{~km}$ por camino mejorado o 3 por tierra hasta la entrada. (2) Sucesión: ya tiene definida la sucesión o tiene deseo de continuar/ Existe la posibilidad de sucesión o no sabe si continuar/ No hay sucesión o la misma pretende abandonar el sistema de producción o no quiere continuar. (3) T: terciario; U: universitario; S: secundario.

\begin{tabular}{|c|c|c|c|c|c|}
\hline Indicador & Componente & \multicolumn{4}{|c|}{ Escala de valoración } \\
\hline \multirow{5}{*}{$\begin{array}{l}\text { Calidad de vida del } \\
\text { entorno }\end{array}$} & Transitabilidad de caminos & (1) & Buena & Regular & Mala \\
\hline & Distancia a centro poblado & $\mathrm{km}$ & $<5$ & $5-10$ & $>10$ \\
\hline & Distancia a centro educativo & $\mathrm{km}$ & $<5$ & $5-10$ & $>10$ \\
\hline & Distancia a centro médico & $\mathrm{km}$ & $<5$ & $5-10$ & $>10$ \\
\hline & Acceso a servicios & & $\begin{array}{l}\text { Buena señal } \\
\text { telefónica e } \\
\text { internet }\end{array}$ & $\begin{array}{l}\text { Poca señal } \\
\text { telefónica }\end{array}$ & $\begin{array}{l}\text { Sin señal } \\
\text { telefónica }\end{array}$ \\
\hline Sucesión & $\begin{array}{l}\text { Predisposición para continuar la } \\
\text { actividad de algún integrante de la } \\
\text { familia }\end{array}$ & $(2)$ & $\begin{array}{c}\text { ya tiene } \\
\text { definida la } \\
\text { sucesión o } \\
\text { tiene deseo de } \\
\text { continuar }\end{array}$ & $\begin{array}{c}\text { Existe la } \\
\text { posibilidad de } \\
\text { sucesión o no } \\
\text { sabe si } \\
\text { continuar }\end{array}$ & $\begin{array}{c}\text { No hay sucesión } \\
\text { o no quiere } \\
\text { continuar }\end{array}$ \\
\hline \multirow{4}{*}{ Formación } & $\begin{array}{c}\text { № cursos realizados en los últimos } \\
\text { tres años }\end{array}$ & № & $2-3$ & 1 & 0 \\
\hline & Nivel de escolaridad alcanzado & (3) & $\mathrm{T} \circ \mathrm{U}$ & $\begin{array}{l}\text { De S completa } \\
\text { a U incompleta }\end{array}$ & $\begin{array}{c}\text { Hasta S } \\
\text { incompleta }\end{array}$ \\
\hline & $\begin{array}{c}\text { № de charlas a las que asistió en los } \\
\text { últimos tres años }\end{array}$ & Frecuencia & $>1$ anual & 1 anual & $<1$ anual \\
\hline & $\begin{array}{l}\text { Capacitaciones realizadas por el } \\
\text { tambero en los últimos tres años }\end{array}$ & № & $2-3$ & 1 & 0 \\
\hline
\end{tabular}

Tabla 3. Indicadores de la dimensión ecológico-ambiental. Referencias. (4) manejo de efluentes: Adecuado: posee un sistema de tratamiento planificado y con las instalaciones y tecnologías apropiadas. Insuficiente: poseen lagunas pero sin el dimensionamiento adecuado y/o no hay una planificación en el manejo y destino final de los efluentes. Sin manejo: rebalse a cuneta/bajo en el tambo o fuera del mismo.

\begin{tabular}{|c|c|c|c|c|c|}
\hline Indicador & Componente & \multicolumn{4}{|c|}{ Escala de valoración } \\
\hline & & Unidad & Verde & Amarillo & Rojo \\
\hline \multirow{2}{*}{$\begin{array}{c}\text { Riesgo de } \\
\text { contaminación de } \\
\text { aguas subterráneas }\end{array}$} & $\begin{array}{l}\text { Distancia de perforación a } \\
\text { laguna de efluentes, a corral } \\
\text { de alimentación y a cámara } \\
\text { séptica }\end{array}$ & $\mathrm{m}$ & $>100$ & $100-50$ & $<50$ \\
\hline & $\begin{array}{l}\text { Ubicación de la perforación } \\
\text { del agua respecto al punto } \\
\text { de concentración de } \\
\text { nutrientes }\end{array}$ & $\mathrm{m}$ & $\begin{array}{c}>100 \text { m aguas } \\
\text { arriba }\end{array}$ & $\begin{array}{l}>100 \mathrm{~m} \text { aguas } \\
\text { abajo a }<50 \\
\text { aguas arriba }\end{array}$ & $\begin{array}{c}<50 \text { m aguas } \\
\text { abajo }\end{array}$ \\
\hline Manejo de efluentes & & (4) & Adecuado & Insuficiente & Sin manejo \\
\hline Emisión de GEI & $\begin{array}{l}\text { Emisión de GEI por kg de } \\
\text { leche corregida }\end{array}$ & $\begin{array}{c}\mathrm{Kg} \mathrm{CO}{ }_{2} \text { eq } / \mathrm{kg} \\
\text { leche corregida }\end{array}$ & $<0,73$ & $0,73-0,97$ & $>0,97$ \\
\hline
\end{tabular}




\section{Resultados y discusión}

En la Figura 1 se observa como el crecimiento del rodeo se tradujo en un aumento de la productividad ajustada a lo largo de los ejercicios productivos analizados.

Se observa un decrecimiento del $25 \%$ y luego de un año hay un aumento abrupto del rodeo del primer al segundo ejercicio analizado, elevándose a un $47 \%$ (esto se produjo por una acumulación de vaquillonas que tuvieron aumentos deficientes de peso diario, y como consecuencia un retraso en la edad al primer servicio, que posteriormente ingresaron todas juntas) y en el tercer período el crecimiento es de un $24 \%$.

El aumento de la productividad a lo largo de los años se puede explicar no solo por el aumento en carga animal, sino por la adecuación de una planificación forrajera que aseguró una adecuada producción y aprovechamiento de las pasturas, además de la utilización de silo de calidad y cantidad para soportar mayores cargas en similar superficie.

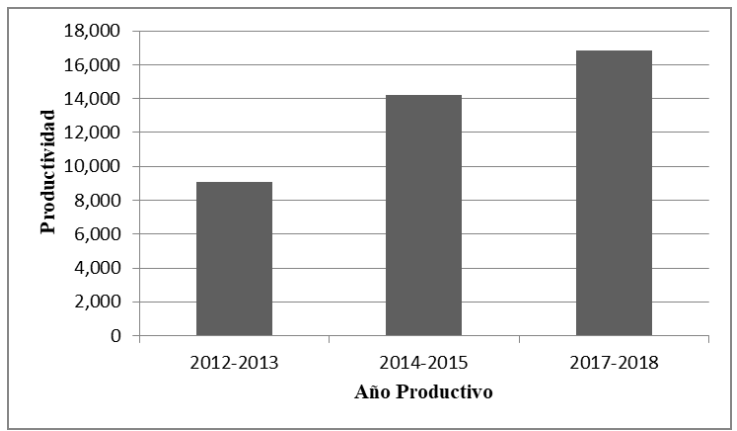

Figura 1. Productividad ajustada (Lts ha $a^{-1}$ año ${ }^{-1}$ ).

La variación abrupta observada en la Figura 2 se debe a un cambio profesional en el manejo de sistema, en el primer ejercicio (2012-2013) se aplicaba un manejo alimentario basado en una dieta dependiente en gran medida de suplementación, en la cual no se aprovechaba el recurso forrajero tanto por falta de manejo como por deficiencias en planeamiento forrajero; en el segundo ejercicio hay una mayor eficiencia en la producción y aprovechamiento de materia seca generada en el establecimiento y en el tercer año aumenta nuevamente el porcentaje de suplementación de concentrado debido a que aumenta la carga animal y el recurso forrajero que ofrece el establecimiento no es suficiente para cubrir los requerimientos del rodeo. La parte técnica en el ejercicio 2012-2013 estaba a cargo de un Ingeniero Agrónomo contratado, el cual tenía un enfoque en sistemas intensivos y con escaso conocimiento sobre estrategias de pastoreo, se planteaban dietas complicadas al personal generando frustración por falta de tiempo para realizar los trabajos, desgaste de maquinaria y gasto de combustible innecesarios. El rodeo se encontraba con bajo estado corporal, no lográndose las producciones individuales propuestas (menores a
$20 \mathrm{~L}$ promedio vaca/año), reduciendo la tasa de concepción anual al $28 \%$ y generando altos costos por litro producido. En los ciclos siguientes, con el hijo del productor incorporado en la empresa, se modifica el equipo de gestión, ya que al incorporar un profesional más, se cambiaron las estrategias de pastoreo, se ajustaron las dietas ofrecidas al rodeo y se organizaron de manera más simple las actividades a realizar dentro del proceso productivo, identificando los puntos críticos de control para lograr mayor impacto productivo reduciendo la cantidad de actividades diarias sobre el personal, articulando de mejor manera las labores y disminuyendo el gasto de combustible y uso de maquinaria destinada a la alimentación del ganado.

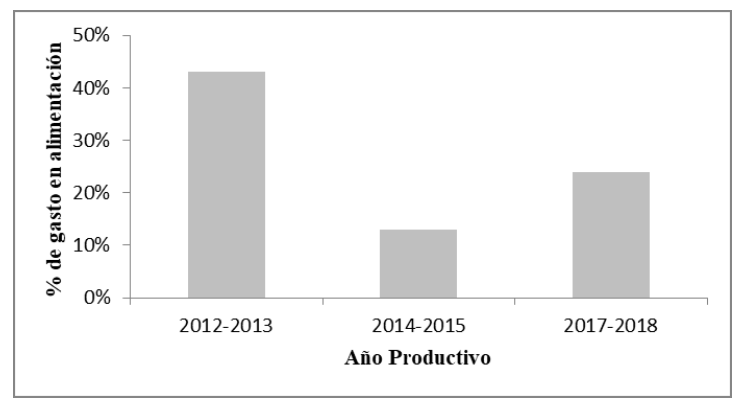

Figura 2: Porcentaje del gasto de alimentación de las VT con relación al ingreso bruto.

Los valores de la Figura 3 muestran la mejora que se observa en el último ciclo analizado. En cuanto a la calidad de vida del entorno el establecimiento se encuentra a $10 \mathrm{~km}$ de ruta pavimentada, centro de atención primaria de salud y escuela primaria. El pueblo es muy pequeño y cuenta con menos de 40 familias y escasas oportunidades de trabajo dentro de la comunidad. La ciudad más cercana con atención médica de calidad y colegios primarios y secundarios se encuentra a $30 \mathrm{~km}$.

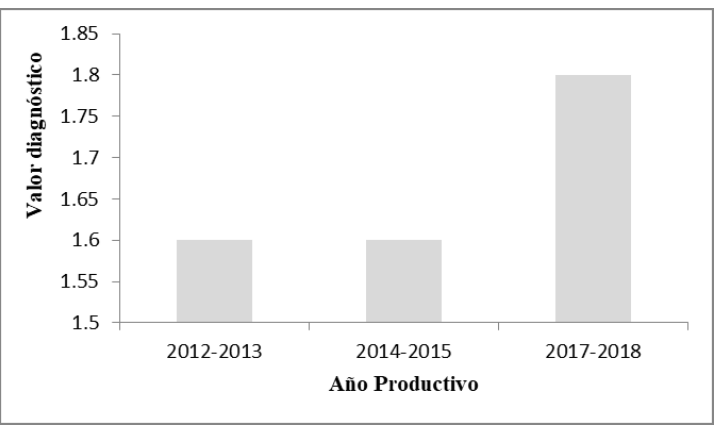

Figura 3: Valores de la calidad del entorno de los tres ciclos analizados.

En cuanto a la energía eléctrica, el establecimiento cuenta con un abastecimiento monofásico lo cual encarece el consumo de energía y genera inestabilidad en la línea sobre todo en verano, debido a que se encuentra al límite de consumo. Esto trae problemas recurrentes en el funcionamiento de motores y equipos de frío. 
En cuanto a la señal de telefonía celular fue mejorando en los últimos tres años, y hace dos años que se cuenta con internet en el establecimiento, lo cual agilizó mucho la comunicación con el personal y el flujo de información para la toma de decisiones. Esta herramienta permite la carga de registros y digitalización de la información a campo por parte del personal.

Si bien la herramienta proporcionada por INTA es útil para dimensionar la infraestructura, el acceso a salud y educación, la misma no arroja resultados en base al confort laboral ni a la calidad de vida la cual es muy subjetiva debido a que depende de la construcción cultural de aquel que habita en un determinado entorno.

En la Figura 4 se observan valores más altos en los dos últimos años productivos, lo cual se relaciona con una mayor certidumbre en la sucesión debido al acompañamiento de dos de los hijos en la empresa y a la conformación de la sociedad a futuro. En la actualidad la empresa se encuentra en un proceso de traspaso de dueño-administrador-fundador a una sociedad donde los cuatro propietarios serán accionistas y tres de los mismos ya forman parte del equipo de gestión. Hay ciertos roles establecidos, hoy los dos hijos mayores cobran un porcentaje de producción preestablecido acorde a el asesoramiento técnico realizado en el establecimiento. El productor no tiene una remuneración por su trabajo administrativo lo cual dificulta tener cierta claridad en la empresa. Aunque se visualizan mejoras, hay un margen posible de progreso al trabajar sobre ciertos factores críticos que tienen impacto en el proceso de sucesión para la continuidad en la empresa. Algunas medidas pueden ser: redefinir la figura del dueño-administrador-fundador, transformando parte de sus retiros en un sueldo acorde a la labor que realiza dentro de la organización; evaluar qué porcentaje de las utilidades se reinvierte en la empresa y qué cantidad de dividendos se redistribuye en los accionistas acorde a su porcentaje de propiedad; establecer una mesa de accionistas, para así mejorar los canales de comunicación e información para con los accionistas y acordar las decisiones en conjunto; seguir explorando opciones de inversión, evaluando posibilidades de participar de las mismas; definir como mínimo reglas en cuanto a la cantidad máxima de deuda a tomar (como porcentaje del activo) y evaluar las tasas máximas a tomar en base a proyectos a llevar a cabo.

Si bien la empresa siempre estuvo a cargo de profesionales, no siempre hubo claridad de qué manera producir ni qué dirección tomar ante distintos escenarios, es por ello, que considera de vital importancia la formación tanto personal como profesional generando valores y conocimiento. Desde sus inicios quienes la integran están en constante aprendizaje, entendiendo a la actividad primaria como un negocio el cual tiene que aportar beneficios en sus tres dimensiones. La posibilidad de seguir en formación constante, relacionada con instituciones, personas idóneas en el sector y fuera del mismo, permite una mayor apertura y visión a la hora de tomar decisiones a futuro.

Los hijos del productor son profesionales que continúan en proceso de formación constante tanto en actividad privada como académica, con el objetivo de tener un enfoque más amplio de la actividad. Están en intercambio constante con profesionales que intervienen en sistemas y procesos. En cuanto al personal que trabaja a diario en el establecimiento, tanto el tambero como su mujer realizaron un curso de tamberos hace tres años (antes de ingresar a la empresa). La pareja más joven (hija y yerno del tambero), realizó durante el año 2019 el programa profesional tambero de INTA.

La empresa realiza reuniones trimestrales para evaluar la satisfacción del personal y monitoreo de objetivos, y los dueños están en constante comunicación con los operarios. Estas mejoras son visualizadas en los resultados que se exponen en Figura 5.

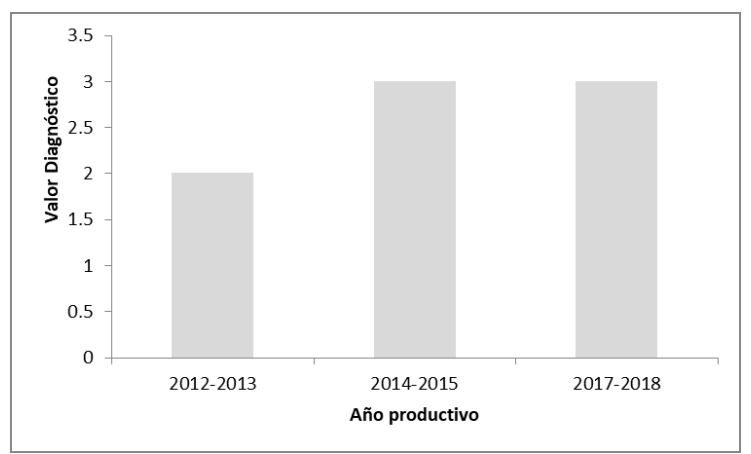

Figura 4: Valores de la sucesión del productor en los tres ciclos analizados.

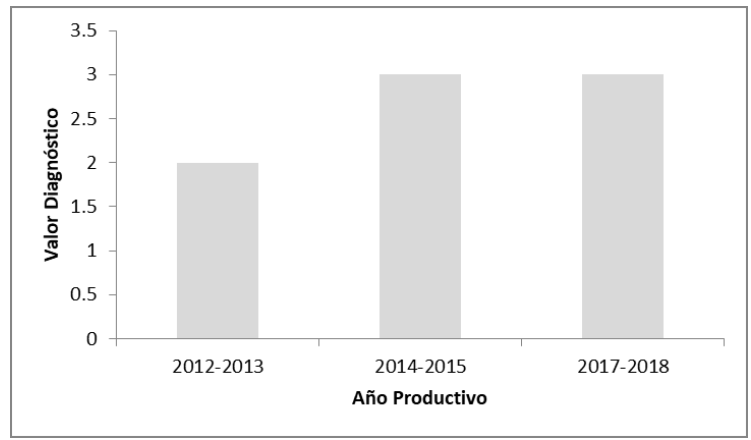

Figura 5: Valores de la formación y estudios en los tres ciclos analizados.

Manejo de efluentes: el establecimiento cuenta con dos fosas interconectadas ( $13 \times 7 \mathrm{~m}$ y $25 \times 7 \mathrm{~m}$ ) ubicadas a cincuenta metros del tambo, en la primera de ellas quedan sedimentadas las excretas más sólidas y sobrepasa a la segunda el contenido líquido. Cada tres meses la primera fosa es mezclada con un sinfín y se aspiran las excretas en una cisterna y se vierte el contenido por los lotes del establecimiento para 
aportar fertilidad al suelo. En la actualidad las fosas no se encuentran impermeabilizadas lo que significa un riesgo en cuanto a la contaminación de napas subterráneas. Es posible que el crecimiento del rodeo haya derivado en que, a igualdad de condiciones en la infraestructura destinada a la gestión de los efluentes, un mayor volumen de excretas producidas tienda a incrementar el riesgo de contaminación subsuperficial.

La herramienta calificó que el manejo de efluentes es adecuado, así como el riesgo de contaminación de agua, aun cuando existen riesgos de contaminación de napas debido a la falta de impermeabilización de las lagunas.

En la Figura 6 se aprecia un descenso en las emisiones de GEI desde los 0,76 kg CO2 eq/ $/ \mathrm{kg}$ leche corregidos del ciclo 2012-13, hasta situarse en el orden de $0,6 \mathrm{~kg} \mathrm{~kg}$ $\mathrm{CO} 2 \mathrm{eq} / \mathrm{kg}$ leche corregidos en los ejercicios posteriores. Dada la expresión del indicador, en cantidades de gases con relación a la producción lechera, es factible proponer que el descenso en las emisiones se vincula con el incremento de la productividad del sistema. La exigua diferencia entre los ejercicios 201415 y 2017-18 puede relacionarse con el incremento en la carga animal, dado durante el último ciclo, especialmente teniendo en cuenta que la mayor parte de las emisiones de GEI en los sistemas lecheros se vinculan con los procesos de fermentación entérica del ganado. La herramienta califica como adecuado el nivel de GEI en los últimos dos ejercicios productivos e insuficiente en el primer año productivo analizado.

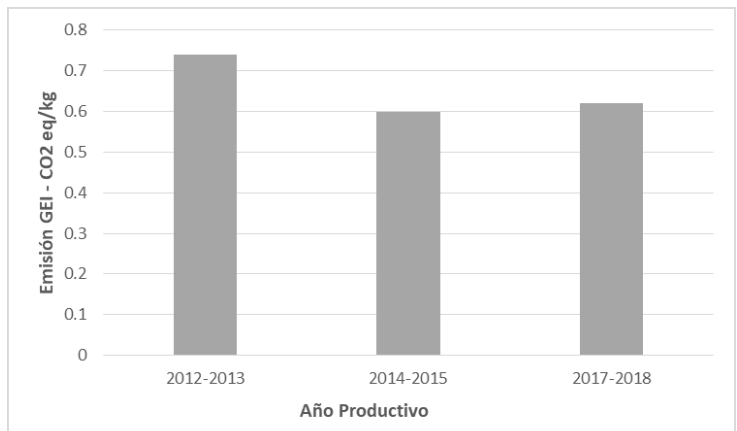

Figura 6. Valores de la emisión de gases efecto invernadero en los tres ciclos analizados.

Tanto el sistema productivo como la empresa han ido creciendo gradualmente a lo largo de los años. La implementación de medidas de manejo de alto impacto y bajo costo en los últimos seis años permitió el crecimiento del rodeo, aumentar un tercio la carga animal en el sistema, para lograr una mayor productividad. Esta mejora crea condiciones para una mayor sustentabilidad en algunos aspectos sociales y ambientales, ya que genera los medios para la formación y profesionalización de los recursos humanos; en tanto que se reducen las emisiones de GEI a la atmósfera.

Si bien la parte técnica en la producción primaria ha sido trabajada en profundidad, todo emprendimiento está construido a partir de valores en común que comparten las personas que lo llevan adelante, los mismos comprenden una base de entendimiento $y$ enmarcan la misión y visión a futuro de la misma. Tener armonía y poder de escucha entre los miembros de la familia es de vital importancia; se debe comprender que la empresa tiene una estructura de gobierno distinta a la de una familia. Es necesario generar encuentros entre los socios fuera de los ámbitos familiares, también se considera de gran utilidad la implementación de asesores externos especializados, los cuales auditan y moderan las reuniones permitiendo que cada uno de los integrantes exprese sus intereses personales. La empresa se encuentra en un proceso de cambio profundo en la cual se están reacomodando los roles para trazar un nuevo horizonte a futuro que contemple los intereses de sus socios.

\section{Agradecimientos}

Esta investigación formó parte del trabajo final de la Especialización en Sistemas de Producción Animal Sustentable de la Facultad de Ciencias Agrarias y Ciencias Veterinarias de la Universidad Nacional de Rosario - Argentina.

\section{Referencias}

Asociación Argentina de Consorcios Regionales de Experimentación Agrícola AACREA. 2011. Calculador de Carbono para tambos. Disponible en: https://www.crea.org.ar/calculador-de-huella-de-carbono-entambo/. Fecha de acceso: Abril de 2021.

Agromercado. 2014. Márgenes de tambo. En Suplemento económico ganadero. Revista № 347, Año 33: 64-65.

Bastos L, Vásquez E, López Y. 2016. Factores que determinan la dinámica de las empresas familiares y su reto con la internacionalización. Revista Lebret 8: 59 - 74

Belausteguigoitía I. 2003. Empresas familiares: su dinámica, equilibrio y consolidación. Ed. Mc Graw-Hill, México.

Centeno A, Almada G. 2019. Tambos flexibles con empresarios resilientes. ¿La clave del éxito? Disponible en: http://www.todoagro.com.ar/noticias/nota.asp?nid=37724. Fecha de acceso: Mayo 2020.

Eurich L, Suero, M. 2012. Sucesión de la empresa familiar agropecuaria: el caso de la empresa "la esperanza". Libro de resúmenes de la XVI Jornadas Nacionales de Extensión Rural, Concordia, Entre Ríos. Argentina. Disponible en: http://www.aader.org.ar/XVI jornada/trabajos/archivos/2012 090 trabajo A atm eurich.pdf

Gómez G. 2013. Dificultades experimentadas por las empresas familiares en los países latinoamericanos: un estudio descriptivo. Family Business. Área INALDE Business School. Inédito.

Giri L. 2015. Reseña: Sistemas Complejos-Rolando García. Preprint depositado en: https://www.researchgate.net/publication/332530781 Sistem 
as complejos-Rolando Garcia-Resena_Giri/. Fecha de acceso: Abril de 2021.

Kaplun C. 2012. La familia en la empresa Parte 1. Universidad de Monterrey, México.

Kets de Vries MF. 1996. Family business: Human dilemmas in the family firm. Thomson Business Press. 287 pp.

Gimenez G, Litwin G, Álvarez H. 2018. Indicadores de sustentabilidad en tambos comerciales de la cuenca lechera central argentina. Avances institucionales en el período 20142018. Serie misceláneas $N^{\circ} 20$. Ediciones INTA. EEA Paraná.

Litwin G, Gimenez G, Alvarez H, Esnaola I, Centeno A, Moretto M, Maekawa M, Butarelli S, Engler P, Spilj G, Almada G, Ferrer J, Tieri M, Charlón V. 2016. Indicadores de sustentabilidad en tambos comerciales de la Cuenca Lechera Pampeana Argentina. Libro de resúmens de la XLVII Reunión Anual de Asociación Anual de Economía Agraria, Mar del Plata, Argentina.

Maza Pereda A, Páez Aragón A. 1991. Causas de mortalidad de la micro y pequeña empresa. IPE, Coparmex y Fundes, México.

Roulet NE. 2013. Lechería: Margen final de un tambo

Disponible en portal Agro Blog.com.ar:

http://agroblog.com.ar/lecheria/lecheria-margen-final-de-untambo.html. Fecha de acceso: Mayo 2020.

Suarez V, Lomello V, Giovannini F, Apto Y, Miranda D. 2019. Particularidades de la gestión de empresas familiares agropecuarias de Argentina. Rev. Cient. FAV-UNRC Ab Intus 4 21-35.

Viglizzo E. 2001. La trampa de Malthus. Agricultura, competitividad y medio ambiente en el siglo XXI. Eudeba, Buenos Aires, Argentina. 133 pp. 